\title{
Illustration of Pre-harvest Peanut Seeds Mycoflora and Mycotoxins
}

\author{
El-Maghraby O. M. O. ${ }^{1}$, Soliman S. A. ${ }^{2}$, El-Sherbeny S. N. ${ }^{3}$, and Ibrahim Y. M. M. ${ }^{4}$ \\ ${ }^{1,2}$ Department of Botany and Microbiology, Faculty of Science, Sohag University, Sohag, Egypt \\ ${ }^{3,4}$ Plant Pathology Institute, Agriculture Research Centre, Giza, Egypt \\ doi:
}

\begin{abstract}
Of pre-harvest peanut seeds (folded seeds), on two isolation media ( $1 \%$ glucose and $1 \%$ cellulose Czapeck's agar), the gross fungal counts on glucose (325 colonies) was higher than on cellulose ( 272 colonies) per 20 samples (each, 10 seeds). Where, the average number of colonies per 10 seeds was higher on glucose (7-32 colonies) than on cellulose (3-21 colonies). Also, $6 \%$ and $7.5 \%$ of the seeds were free of filamentous fungi on the two isolation media, respectively. Using seed-plate method and without surface disinfection, 7 species of 5 genera were isolated and identified from peanut seeds on glucose ( 6 species of 5 genera) and cellulose (7 of 5) Czapeck's agar at $18 \pm 10 \mathrm{C}$. Of isolated genera, Aspergillus was superior in count (56\% and $53.3 \%$ of fungal count, respectively) and frequency ( $85 \%$ and $90 \%$ of samples, respectively) on the isolation media.
\end{abstract}

KEYWORDS

Peanut,

Seeds,

Fungi,

Mycotoxins.

\section{CORRESPONDING}

\section{AUTHOR}

Othman M. El-Maghraby osmanelmaghraaby@yahoo .com

Of the genus, $A$. flavus had the highest count (34.5\% and $29.4 \%$ ) and frequency (each, $80 \%)$. $A$. niger was less in count $(21 \%$ and $17.4 \%)$ and in occurrence (each, $55 \%$ ) on the two media. Two species (Fusarium oxysporum and Penicillium citrinum) were detected in moderate frequencies (40 $\%$ and $30 \%$ of the samples on the two media, respectively). F. oxysporum had the higher count (14.2 $\%$ and $16.2 \%$ on the two media) compared with $P$. citrinum (each, $11.4 \%$ ). Cladosporium cladosporioides was low in occurrence (each, $20 \%$ of the samples) with counts $12 \%$ and $11.4 \%$. A. fumigatus appeared on cellulose medium only whereas, Nectria haematococca on glucose only. Based on biological assay (brine shrimp test) and chemical detection (TLC and UV spectra), the ethyl acetate extracts of pre-harvest peanut seeds (approx. 15-20 days before harvest) proved to be non-toxic and mycotoxin free.

\section{INTRODUCTION}

Peanuts have been grown and consumed for approximately 4000 years. They are an important cash crop, with current production of over 40 million metric tons annually. Recent cultivation of high-oleic varieties has recently increased to improve stability and health benefits. Additionally, peanuts are an important source of nutrition worldwide, as they are rich source of proteins, fibers, micronutrients and phytochemicals.
Moderate intake has not been associated with weight gain, but is associated with glucose tolerance and reduced cardiovascular disease rick (Jones et al. 2016).

Different studies of developing countries showed that the threat of fungi and mycotoxins contamination of foods and feeds resulting in human and livestock poisoning is really a major problem (Wild 2007; Shehpard 2008). Soil is a 
source of primary inoculum of Aspergillus flavus and $A$. parasiticus, fungi that produce highly carcinogenic aflatoxins in peanut. Aflatoxigenic fungi commonly invade peanut seeds during maturation, and the highest concentrations of aflatoxins are found in damage seeds. Aerial crops such as corn and cotton seeds are infected predominantly with $A$. flavus, whereas peanut with their subterranean growth habit are invaded by both A. flavus and A. parasiticus (Horn 2003).

Toxin-producing moulds may invade plant material in the field before harvest, during postharvest handling, storage and during processing into food and feed products. Thus, toxigenic fungi have been classified into two groups (i) field fungi; (ii) storage fungi. Fusarium species are ubiquitous soil organisms which may infect cereals directly in the field, thereby producing fumonisins, trichothecenes and zearalenone (depending on the species) during growth, ripening of grains and at harvest (Jouany 2007).

The present work was designed for studying the filamentous fungi associated with peanut seeds before harvest (approx. 15-20 days) in addition to natural occurrence of mycotoxins of peanut seeds.

\section{MATERIALS AND METHODS}

\section{Collection of pre-harvest peanut fruits}

Pre-harvest (approx. 15-20 days before harvest) peanut fruits (20 samples) from farmer's field in Upper Egypt (5 governorates, El-Giza, Assiut, Sohag, Qena and Aswan) were collected. The samples were placed in sample bags, sealed and placed in other bags which were also sealed. The samples were kept cool during transfer (3-50C) to the laboratory.

\section{Isolation and identification of mycoflora}

In laboratory, the peanut fruits were subjected to series of washing with sterile dist. water. The seeds were unfolded, divided to two segments (two cotyledons), dried between sterilized filter paper. The segments ( 10 cotyledons) were inserted on the surface of two isolation (1\% glucose and $1 \%$ cellulose Czapeck's agar) media (2 segments per Petri dish) supplemented by chloramephenicol $1 / 30000$ as reported by Al-Doory (1980). The dishes were incubated at 18 $\pm 10 \mathrm{C}$ for 7-10 days The growing colonies were examined, counted (per segment), isolated and identified based on morphological features according the keys of Raper and Thom 1949; Raper \& Fennell 1965; Booth 1977; Nelson et al. 1983 and Moubasher 1993.

\section{Extraction of mycotoxins}

Twenty-five $g$ of peanut seeds were continuously defatted by extraction with $\mathrm{n}$-hexane for $10 \mathrm{~h}$ using soxhlet-type extractor. The defatted residue was reextracted by ethyl acetate $(75 \mathrm{ml})$ in a $250 \mathrm{ml}$ Erlenmeyer flask, shaken using a rotary shaker (200 rpm, $24 \mathrm{~h}$ ) and filtered through Whatman No. 1 filter paper. Then, the residue was washed twice with ethyl acetate (each, $25 \mathrm{ml}$ ). The ethyl acetate extracts were combined, dried over anhydrous sodium sulphate $(5 \mathrm{~g})$, concentrated in vacuo, transferred to glass vial and evaporated in air.

\section{Clean up of crude extract:-}

For cleanup of crude extract, a silica gel column $(14 \times 0.4 \mathrm{~cm})$ containing $2.5 \mathrm{~g}$ Kiesel gel 60, 70/230 (Merck) was used as follows: Aflatoxins, ochratoxins A \& B sterigmatocystin, and zearalenone were cleaned up according to AOAC (1984), whereas trichothecenes were processed according Jarvis et al. (1986).

\section{Biological assay:-}

For bioassay of mycotoxins, brine shrimp (Artemia salina L.) larvae ( $3^{\text {th }}$ nauplii) were used based on the korpinen (1974) method. A filter paper disc $\left(1 \mathrm{~cm}^{2}\right)$ was saturated with $50 \mu \mathrm{g}$ of crude extracts, air dried and the discs were added to sea water $(2 \mathrm{ml})$ containing the larvae (40-60 
larvae). The mortality of the larvae was estimated after $16 \mathrm{~h}$.

\section{Chemical detection of mycotoxin}

Thin layer chromatography (TLC) was carried out on silica gel plates (Merck). Aflatoxins B1, B2, G1 \& G2, ochratoxins A \& B, sterigmatocystin, zearalenone, T-2 toxin, diacetoxyscirpenol (DAS) were applied as standards. The developing solvent system was methanol-chloroform ( $\mathrm{v} / \mathrm{v}, 3: 97)$, and the developing plates were viewed under 254 and $366 \mathrm{~nm}$ irradiation or spread with reagents (AOAC 1984 and Jarvis et al. 1986).

Spectrophotometer (Cecil model 703) was used for detection the mycotoxins using the molecular coefficient of 21.800 at 254 and $366 \mathrm{~nm}$. The fluorescent zones, including standards were removed from TLC plates and dissolved in methanol-chloroform (v/v, 5: 95).

\section{RESULTS}

\section{Filamentous Fungi of Pre-harvest Peanut Seeds}

Of filamentous fungi associated with pre harvest peanut seeds (20 samples) on two isolation media (1\% glucose and $1 \%$ cellulose Czapeck's agar) revealed that, glucophilic (325 colonies/200 cotyledons) were higher than cellulose-decomposer (272 colonies) fungi. Where, the number of colonies per sample fluctuated between 7-32 and 321 colonies on glucose and cellulose agar, respectively. On other side, the percentage of uninfected (health) seeds was $6 \%$ and $7.5 \%$ on the two isolation media, respectively (Table 1).

Table (1) Total count (TC) calculated per 20 peanut seed samples (each, 10 cotyledons), total count percentage per gross count (TC \%), number of cases of isolation (NCI) and occurrence remarks (OR) of fungal genera and species isolated on glucose- and cellulose-Czapeck's agar at $18 \pm 10 \mathrm{C}$.

\begin{tabular}{|c|c|c|c|c|c|c|}
\hline \multirow{2}{*}{ Genera and species } & \multicolumn{3}{|c|}{ Glucose-Czapek,s agar } & \multicolumn{3}{|c|}{ Cellulose-Czapek,s agar } \\
\hline & TC & TC \% & NCI \& OR & TC & TC\% & NCI \& OR \\
\hline Aspergillus & 182 & $56 \%$ & $17 \mathrm{H}$ & 145 & $53.3 \%$ & $18 \mathrm{H}$ \\
\hline A. flavus Link & 112 & $34.5 \%$ & $16 \mathrm{H}$ & 80 & $29.4 \%$ & $16 \mathrm{H}$ \\
\hline A. niger Van Teighem & 70 & $21.5 \%$ & $11 \mathrm{H}$ & 48 & $17.4 \%$ & $11 \mathrm{H}$ \\
\hline A. fumigatus Fresenius & - & - & - & 17 & $6.4 \%$ & $6 \mathrm{M}$ \\
\hline Fusarium oxysporum Schlecht ex Fr. & 46 & $14.2 \%$ & $8 \mathrm{M}$ & 44 & $16.2 \%$ & $8 \mathrm{M}$ \\
\hline Penicillium citrinum Thom & 37 & $11.4 \%$ & $6 \mathrm{M}$ & 31 & $11.4 \%$ & $6 \mathrm{M}$ \\
\hline Cladosporium cladosporioies $(\mathrm{Fr})$ de Vries & 39 & $12 \%$ & $4 \mathrm{~L}$ & 31 & $11.4 \%$ & $4 \mathrm{~L}$ \\
\hline Nectria haematococca Berkely \& Brown & 21 & $6.5 \%$ & $-3 \mathrm{~L}$ & -21 & $7.7 \%$ & $3 \mathrm{~L}$ \\
\hline Gross total count & \multicolumn{3}{|c|}{325} & \multicolumn{3}{|c|}{272} \\
\hline Number of fungal species and genera & \multicolumn{3}{|c|}{6 species $/ 5$ genera } & \multicolumn{3}{|c|}{7 species $/ 5$ genera } \\
\hline Number of colonies (per 10 cotyledons) & \multicolumn{3}{|c|}{ 7-32 colonies } & \multicolumn{3}{|c|}{ 3-21 colonies } \\
\hline Percentage of healthy seeds & \multicolumn{3}{|c|}{$6 \%$} & \multicolumn{3}{|c|}{$7.5 \%$} \\
\hline
\end{tabular}

Concerning to isolated fungal species and their genera, a total of 7 species of 5 genera were isolated and identified on glucose (6 species of 5 genera) and cellulose (7 of 5) agar media. Aspergillus was quite the dominant genus on the two isolation media $(56 \% \& 53.3 \%$ of gross fungal count, respectively).
Of the genus, $A$. flavus was superior in counts $(34.5$ $\%$ and $29.4 \%$ of gross counts on the two isolation media, respectively) and frequencies ( $80 \%$ of the samples on both media), followed by $A$. niger which was less in counts $(21.1 \%$ and $17.4 \%$ of total fungi, respectively) and frequencies (each, 55 $\%$ of samples). A. fumigatus was only observed on 
cellulose medium in less count $(6.3 \%)$ and frequency $(30 \%)$. Of moderate frequency of occurrence, two species of two genera (Fusarium oxysporum and Penicillium citrinum) were isolated and identified on the two media tested. $F$. oxysporum had the higher counts $(14.2 \%$ and 16.2 $\%$ of gross fungi) with the same frequency (each, $40 \%$ of samples), whereas $P$. citrinum was less in counts (each, $11.4 \%$ ) and occurrence (each, $30 \%$ ). Finally, Cladosporium cladosporioides and Nectria haematococca had low frequencies $(20 \% \& 15 \%$ of peanut seed samples) and the counts were higher of the first species $(12 \% \& 11.4 \%$ of gross fungal counts on the two media) than the second $(6.5 \% \&$ $7.7 \%$, respectively).

\section{Mycotoxins Analysis}

Of pre-harvest peanut seeds (15-20 days before harvest), the ethyl acetate extracts (20 samples, each $25 \mathrm{~g}$ ) after de-fatted by n-hexane proved that, all samples tested were non-toxic (less than $25 \%$ dead larvae) to brine shrimp (Artemia salina L.) larvae ( $3^{\text {th }}$ stage of nauplii). Based on chemical analysis (Thin layer chromatography "TLC" and UV spectrophotometric analyses), there is no mycotoxins could be detected.

\section{DISCUSSION}

Of isolated filamentous fungi with regarding the total fungal counts and detected genera and species on two isolation media (glucose- and celluloseCzapeck's agar), the gross fungal count on glucose was more than on cellulose, whereas the number of species on cellulose was higher compared with glucose. These results accepted with El-Sherbeny et al. (2020) through isolation filamentous fungi from damping off and wilting off diseases of peanut plants. Also, glucose is easy and quickly utilizing mono-saccharides in glycolysis process than other carbohydrates (Sequeira et al. 2019). Therefore, cellulose (polysaccharide) especially with $\mathrm{pH} 8$ has the ability to restrict the growth of heavy sporulating filamentous fungi to give good chance for growing non-heavy sporulating fungi (El-Maghraby \& El-Maraghy 1988).

Based on identified fungal species, Aspergillus flavus and $A$. niger were quite the most dominant based on frequency and count. In this respect, A. flavus was amongst toxigenic storage fungi (Horn 2003), the previous two species in addition $\mathbf{A}$. fumigatus were the commonest species in stored peanut seeds (El-Maghraby \& El-Maraghy 1987, 1988) and the only species isolated from peanut plants (El-Khadem 1975). Two Fusarium species namely, $F$. oxysporum and $F$. solani (Nectria haematococca) were detected in moderate and low frequencies with moderate and low counts, respectively. Concerning these results, Fusarium amongst toxigenic field fungi (Horn 2003), widely detected in Egyptian damping off and wilting off diseases of Egyptian peanut plants (El-Sherbeny 2020). Whereas, six Fusarium spp. were isolated and identified from stored peanut seeds (40 samples) as reported by El-Maghraby \& ElMaraghy (1988). The remaining two species, Penicillium citrinum and Cladosporium cladosporioides, were observed in moderate and low frequencies of occurrence, respectively with moderate counts. In this respect, the previous two species disappeared in damping off and wilting off diseases of peanut plants (El-Sherbeny et al. 2020), whereas widely detected as endophytes of peanut shoots (leaves and stems) as reported by ElMaghraby et al. (2009). The authors in aware, the source of underground fruits filaments fungi related mainly to the soil and less to endophytic fungi which transfer from shoot to root.

Concerning to toxicity and mycotoxin of preharvest peanut seeds, all samples tested were nontoxic and free of mycotoxins. But, of stored peanut seeds in Egypt, $47 \%$ of chloroform extracts proved to be toxic to brine shrimp larvae (El-Maghraby \& El-Maraghy 1987) and $20 \%, 10 \%$ and $0 \%$ of untreated, roasted and roasted with salts were toxic to 
the larvae (Youssef $\boldsymbol{e t}$ al. 2009). Aflatoxins (B1, B2, G1 \& G2), trichothecenes (T-2 toxin \& diacetoxysripenol) were superior in occurrence followed by, zearalenone and citrinin which were detected in toxigenic samples. The aflatoxins are a group of toxic and carcinogenic polyketide secondary metabolites, which are produced by strains of Aspergillus flavus group (Ito et al. 2001). The international Agency for Research on Cancer (IARC) has classified aflatoxins as a group I carcinogen affected liver (IARC 1993), contaminated various agricultural commodities with highest risk of oil seeds and/or grains including corn, peanut, cottonseed, nut (Pitter, 1998). Trichothecene toxins are a group of related mycotoxins that possess a tetracyclic 12, 13epoxytrichothec-9-ene skeleton. These biologically active metabolites are associated predominantly with Fusarium species, but they also are produced by several other fungi including Myrothecium, Stachybotrys, Trichothecium, Acremonium (Cephalosporium), Cylindrocapon and Trichoderma (Ueno 1983). Although more than 190 derivatives of trichothecenes have been detected in the laboratories, only 11 (T-2 toxin, Ht-2 toxin, AcHT2 toxin, T-2 tetraol, diacetoxyscirpenol, triacetoxyscirpenol, nivalenol, deoxynivalenol, fusarin $\mathrm{C}$, fusarenone $\mathrm{X}$, and neosolaniol) have been detected occurring naturally in feedstuffs, grains and seeds (Ueno 1983; El-Magraby et al. 1995; El-Maghraby 1996; Desjardins 2006; McCormick et al. 2011). Zearalenone is an important mycotoxin, has estrogenic properties, as it causes a variety of reproductive disorder in female farm animals. It is found in maize as well as small grains and mainly produced by Fusarium spp. associated with deoxynivalenol (Pitt, 2014), T2 toxin and diacetoxyscirpenol (El-Maghraby \& El-Maraghy 1987, 1988). Citrinin (one derivative) is nephrotoxic mycotoxin produced by several strains belonging to Aspergillus, Penicillium and Monoascus. It contaminates various commodities of plant origin, cereal is particular and it usually found together with another nephrotoxic mycotoxin, ochratoxin A (Doughari 2015). The toxin is not widely detected in Egyptian cereal grains or oil seeds (El-Maghraby \& El-Maraghy 1987; El-Magraby et al. 1995; El-Maghraby 1996; Youssef et al. 2009).

In conclusion, of underground peanut fruits (seeds), there are several aspects:

- There is no specific filamentous fungus of peanut seeds.

- The isolated fungi were mainly amongst soil fungi and less to endophytes of peanut (leaf and stem) plant.

- The presence of toxigenic fungi is not meaning the presence of their mycotoxins.

\section{REFERENCES}

Al-Doory Y. (1980). Laboratory Medical Mycology. Lea and Febiger, Philadelphia, USA, pp 410.

AOAC (1984). Association of Official Analytical Chemists. Official Methods of analysis $14^{\text {th }}$ ed., Washington DC, USA, 249-255.

Booth C. (1977). Fusarium, Laboratory Guide to the identification of the major species. IMI, Ferry Lane, Kew, Surrey, England, pp 58.

Desjardins A. E. (2006). Fusarium Mycotoxins, Chemistry, Genetics and Biology. APS Press. Eagan, MN, USA.

Doughari J. H. (2015). The occurrence, properties and significant of citrinin mycotoxin. Journal of Plant Pathology and Microbiology, 6: 1116.

El-Khadem M. (1975): Pre- and post-emergence losses of peanut caused by Aspergillus flavus Link. Tech. Mikrobiol. 130 (3): 245-250.

El-Maghraby O. M. O. (1996). Mycotoxins and mycoflora of rice in Egypt with special reference to trichothecenes production and control. Journal of Natural Toxins, 5(1): 4959. 
El-Maghraby O. M. O. and El-Maraghy S. S. M. (1987). Mycoflora and mycotoxins of peanut (Arachis Hypogeae L.) seeds in Egypt. 1Sugar fungi and natural occurrence of mycotoxins. Mycopathologia, 98: 165-170.

El-Maghraby O. M. O. and El-Maraghy S. S. M. (1988). Mycoflora and mycotoxins of peanut (Arachis hypogaea L.) seeds in Egypt. 2Cellulose-decomposing and mycotoxinproducing fungi. Mycopathologia, 104: 19-24.

El-Maghraby O. M. O., El-Kady I. A. and Samia S. (1995). Mycoflora and Fusarium toxins of three types of corn grains in Egypt with special reference to trichothecenes toxins. Microbiological Research, 150: 225-232.

El-Maghraby O. M. O., Youssef M. S. and Ibrahim, Y.M. (2009): Aflatoxins effect on fungal populations of soil, root and leaf surface of peanut plants in the field with special reference to aflatoxins biodegradation. Research Journal of Environmental toxicology, 3(1): 36-49.

El-Sherbeny S. N., El-Maghraby O. M. O., Soliman S. A. and Ibrahim Y. M. M. (2020). Pathogenic and/or saprophytic fungi of wilted peanut seedling and their role in pathogenicity of peanut seeds. Journal of Basic \& Applied Mycology, 11: 99-105.

Horn B. W. (2003). Ecology and population biology of aflatoxigenic fungi in soil. Journal of Toxicology: Toxin Reviews, 22: 351-379.

Ito Y., Peterson S. W., Wicklow D. T. and Goto T. (2001). Aspergillus pseudotamarii, a new aflatoxin producing species in Aspergillus section flavi, Mycological Research, 105: 233239.

IARC "International Agency for Research on Cancer" (1993). Monograph on evaluation of carcinogenic risk to humans, Lyon, France, vol. 56.

Jarvis B. B., Lee Y. W., Comezoglu S. N. and Tatawara C. S. (1986). Trichothecenes production by Stachybotrys atra from Eastern Europe. Applied Environmental Microbiology 51: 915-918.

Jones J. B., Barkely N. A., Simpson, C. E. and Mattes R. D. (2016). Reference Module in Food Science from Encyclopedia of Food and Health, Peanut. pp 277-282.

Jouany J. P. (2007). Methods of presenting decontamination and minimizing the toxicity of mycotoxins in feeds. Animal Feed Science and Technology, 137: 342-362.

Koprinen E. L. (1974). Studies on Stachybotrys alternans 1-Comparison of rabbit skin, mouse fibroblast culture brine shrimp as detectors of Stachybotrys toxins. Acta pathologica et microbiologica Scandinavica, section B: Microbiology and immunology, 82: 465-469.

McCormick S. P., Stanley A. M., Stover N. A. and Alexander N. J. (2011). Trichothecenes from simple to complex mycotoxins. Toxins, (7): 802-814.

Moubasher A. H. (1993). Soil Fungi in Qater and Other Arab Countries. Center for Scientific and Applied Research. University of Qater, Qater, pp 566.

Nelson P. E., Toussoun T. A. and Marasas W. F. O. (1983). Fusarium species, An Illustration of Manual for Identification. The Pennsylvania State University Press. PN, USA, pp 203.

Pitt J. I. (2014). Mycotoxin zearalenone. Food Science, 2: 313-314.

Pitter A. (1998). Natural occurrence of mycotoxins in foods and feeds. Review. Med. Vet. 149: 479-492.

Raper K. P. and Fennell D. I. (1965). The Genus Aspergillus. Williams \& Wilkins, Baltimore, MD, USA, pp 686.

Raper K. P. and Thom C. (1949). A Manual of Penicillium. Williams \& Wilkins, Baltimore, MD, USA, pp 875. 
Sequeira M. G., Kapoor, K. K., Yadov K. S. and Tauro P. (2019), An Introduction of Microbiology. New Agency International Limited, pp 944.

Shehpard G. C. (2008). Impact of mycotoxins on human health in developing countries. Food Additives \& Contaminants, 25: 146-151.

Ueno Y. (1983). Trichothecenes, Chemical, Biological and Toxicological Aspects. Elsevier, Amsterdam, New York, pp 223.
Youssef M. S., El-Maghraby O. M. O. and Ibrahim Y. M. (2009). Mycobiota and mycotoxins of Egyptian peanut (Arachis hypogeae L.) seeds. International Journal of Botany, 18 (11): 1-12.

Wild C. P. (2007). Aflatoxin exposure in developing countries, the critical interface of agriculture and health. Food and Nutrition Bulletin, 28: 372-380. 